\title{
Development and Evaluation of a Web-based Chinese Poetry Learning Support System
}

\author{
Jili Liao \\ School of Chinese Language and Literature \\ Central China Normal University \\ Wuhan, China \\ liaojili@126.com
}

\author{
Yong Gan \\ Department of Chinese language and literature \\ Zhongnan University of Economics and Law \\ Wuhan, China \\ ganyong2002@sina.com
}

\begin{abstract}
Web-based learning has become an important way to enhance teaching and learning. There are a lot of foreigners learning Chinese language and culture via various ways in the world. To help them learn better, a Web-based Chinese poetry learning support system named 'e-Tang' has been designed and implemented. This system provides abundant learning materials and effective interactive tools, and a fuzzy control algorithm is employed to arrange different learning courses for different learners. With this system, learners can study about Chinese poetry, do exercises, and communicate with others, etc. After using this system, the learners' attitudes toward 'e-Tang' were investigated online. The results show that most learners have positive attitudes toward this system, and they believe that the 'e-Tang' system can effectively support and promote them when learning Chinese poetry.
\end{abstract}

Keywords-Chinese poetry; Web-based system; Web technology; Fuzzy control algorithm

\section{INTRODUCTION}

Using Web technology for instruction has expanded rapidly in the past decade. Nowadays, there are many Webbased systems where online courses can be created and accessed in very easy ways. Therefore, more and more online lectures created for self-learning will be a trend. In this paper, we describe an attempt to provide a Web-based system called 'e-Tang' which enables learners to study knowledge about Chinese poetry through Web pages. We have utilized this system as an e-learning course to study its usefulness and effectiveness in Chinese poetry learning.

\section{LITERATURE REVIEW}

\section{A. Reading Using the Web}

With the popularity of Internet, more and more people have become accustomed to reading using the Web. An investigation concerning the reading medium was conducted on 37 foreign students at Central China Normal University in May 2012. As a result, 35 respondents stated that the Web is a necessary existence for them. Nineteen respondents $(51 \%)$ said they read news, blogs, and books using the Web everyday. And 12 respondents (32\%) did these activities every two or three days. Besides the purpose of entertainment, reading using the Web can also be applied to the purpose of learning. Thirteen respondents (35\%) said they collect information from the Web in order to write their reports every one to three days, and 19 respondents $(51 \%)$ said they do it every week. It can be seen from this investigation that reading using the Web has become popular, and offers the practicable possibility for Web-based learning.

\section{B. Chinese Poetry Learning of Foreigners}

As one of the representatives of Chinese classical literature and culture, Chinese poetry received considerable acclaim and possessed many learners in the world. In order to investigate the status of Chinese poetry learning of foreigners, a series of investigations were conducted in our previous research [4] in 2007.

Firstly, Japanese students, For example, should begin to study Chinese poetry from junior/senior high school according to curriculum guidelines of Japan. Textbooks used in General Japanese, which is a compulsory subject in Japanese senior high school, were reviewed by us. It was found that Chinese poetry was a part of 20 textbooks made by 10 publishing companies. The average number of Chinese poems in each textbook is 8. Excluding repeated poems, 38 poems by 18 poets are embodied in these textbooks. Secondly, a questionnaire about Chinese poetry learning was given to 29 Japanese students and most of the students $(65 \%)$ said they think that Chinese poetry is meaningful and elegant in rhythm. These investigations above show that Chinese poetry is an indispensable learning material which Japanese learners can enjoy.

Moreover, there are more and more foreign learners who begin to learn Chinese nowadays, but most of them feel that the pronunciation of Chinese is the first difficulty they meet. Under these circumstances, Chinese poetry is an appropriate exercise material to study Chinese PinYin for Chinese beginners, because it has the catchy sound of a simple, repetitious rhyme. Actually, there are several Chinese learning textbooks which include Chinese poetry overseas, such as [1] and [3].

\section{Chinese poetry learning sites}

With the development of Web technologies, the number of Chinese poetry learning sites has increased. Generally speaking, those sites include poems, word annotations, and analyses of poems. However, those sites are little more than 
electronic versions of Chinese poetry anthologies. The limitations of current Chinese poetry sites are as follows:

- It is possible for learners to comprehend the meaning of poems and even recite a few poems after learning via the current sites. But it is impossible for them to comprehend Chinese poetry as a whole and know the reason why Chinese poetry is one of the representatives of Chinese classical literature and culture because the current sites are never or rarely combine the background of the Tang Dynasty to investigate the development of Chinese poetry, or uncover the unique rhythmical characteristics of Chinese poetry.

- The current Chinese poetry sites are mostly constructed as personal hobbies, not for educational purposes. Therefore, different knowledge nodes are not designed in accordance with the level of learners, and no exercises or tests are provided for assessing the learning of Chinese poetry.

- At present, there is little special site for Chinese poetry learning aimed at foreigners, and almost no Chinese poetry database for foreign learners.

The main purposes of this research were to design and implement a Chinese poetry learning support system, named 'e-Tang', for foreign learners, and to investigate its influence on Chinese poetry learning. The main subjects of this system include those who are interested in Chinese poetry, and Chinese beginners who want to pronounce Chinese better.

\section{RESEARCH DESIGN}

\section{A. Objectives}

For the sake of foreign learners who are interested in Chinese poetry, we prepared abundant learning materials and effective interactive tools, and uploaded them to the Web. The learners were invited to read the lecture materials about Chinese poetry at any moment everywhere through accessing those Web pages, and to complete related exams and a questionnaire later. From February 2013 to April 2013, 22 learners participated in Chinese poetry learning using the 'e-Tang' system. This research intends to explore how learners use options provided by the system on the Web, and to investigate their attitudes toward the system.

\section{B. Components}

As Fig. 1 shows, the framework of 'e-Tang' consists of three main components: 'e-Tang' Database, WWW Server, and Client Side.

The 'e-Tang' Database records correlated data for Chinese poetry learning, including learners' information, Tang poems, questions concerning exercises/tests, and communication topics. Once learners have a behavior such as browsing, searching, testing, or communicating, 'e-Tang' is capable of distilling the corresponding data from the 'eTang' Database and sending them back to the learners. Moreover, the results of their actions, such as Web logs, exercise/test scores, and the topics of communication are saved in the corresponding database.

The WWW Server receives, deposits, and dispatches those events to the Client Side. When a user accesses this system through a Web browser and requests a specific lecture, all correlated contents are dispatched and transmitted to the client side.

The Client Side displays the interface for learners to join in Chinese poetry learning. When a user accesses this system, the prepared lectures appear on the user's screen.

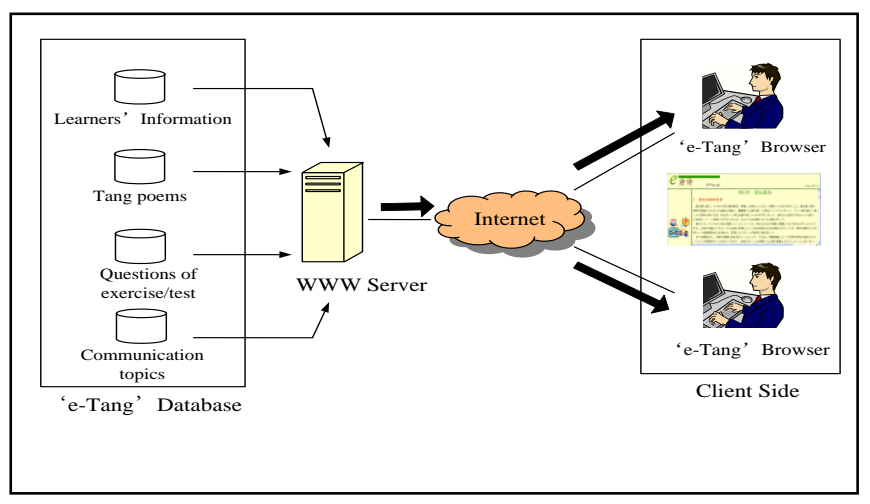

Figure 1. The framework of 'e-Tang'

\section{Procedure}

There are some materials for Chinese poetry learning on the Web. However, as practical applications, those contents are unsuitable because they are too simple and not comprehensive. The textbook of 'e-Tang' has been compiled by collecting and amending previous documents so as to make it more comprehensive. Now there are 4 lectures in the textbook, and each lecture consists of three parts: An introduction to Chinese poetry, Chinese poetry appreciation, and exercises (shown in Table I). The level of difficulty of the lectures is divided into five ranks. Rank 1 is the easiest and Rank 5 is the most difficult. All of the lectures are written in English.

TABLE I. CONTENTS OF TEXTBOOK IN 'E-TANG’

\begin{tabular}{|c|l|l|c|}
\hline \multirow{2}{*}{ Lecture } & \multicolumn{3}{|c|}{ Contents } \\
\cline { 2 - 4 } & $\begin{array}{l}\text { An Introduction } \\
\text { to Chinese poetry }\end{array}$ & \multicolumn{1}{|c|}{$\begin{array}{c}\text { Chinese poetry } \\
\text { Appreciation }\end{array}$} & Exercises \\
\hline 1 & $\begin{array}{l}\text { Tang dynasty and } \\
\text { Chinese poetry }\end{array}$ & $\begin{array}{l}\text { Departure from } \\
\text { Baidicheng at dawn } \\
\text { (Li Bai) }\end{array}$ & exercise 1 \\
\hline 2 & $\begin{array}{l}\text { Genre of Chinese } \\
\text { poetry }\end{array}$ & A spring view (Du Fu) & exercise 2 \\
\hline 3 & $\begin{array}{l}\text { Rhyme and } \\
\text { rhythm }\end{array}$ & $\begin{array}{l}\text { At heron lodge (Wang } \\
\text { Zhihuan) }\end{array}$ & exercise 3 \\
\hline 4 & $\begin{array}{l}\text { Combination of } \\
\text { level and oblique } \\
\text { tones }\end{array}$ & $\begin{array}{l}\text { Remembering Yuan } \\
\text { jiu on night duty in } \\
\text { palace on August 15 } \\
\text { (Bai Juyi) }\end{array}$ & exercise 4 \\
\hline
\end{tabular}

At present, learners can access the 'e-Tang' system and study Chinese poetry at their own pace. Considering the point of view of a learner, the procedures for Chinese poetry learning are described below. 
- First, access the homepage of 'e-Tang' and register.

- Log in by inputting username and password.

- Pre-test. In order to measure prior knowledge of the students, a pre-test which is composed of 10 questions is held at the beginning of learning.

- Individual reading and group communication. Learners who enter this system were asked to read online materials individually. The reading materials of 'e-Tang' consist of An Introduction to Chinese poetry, Chinese poetry Appreciation, and 300 Tang Poems. While reading the materials, learners are allowed to click the communication button and to communicate with other learners using a chat room, BBS, and e-mail.

- Exercise and post-test. At the end of each lecture, an exercise which includes 5 questions is held to measure whether the learners have mastered knowledge about Chinese poetry. When learners submit the answers online, a score and explanation of answers are shown immediately. In addition, a post-test, which is the same as the pre-test, is held after all the lectures are finished. A comparison between the pre-test and post-test in scores enables the learning efficiency to be assessed.

- Questionnaire. A questionnaire devised by the researchers was given to the learners to evaluate their attitudes toward 'e-Tang'.

After the procedures above, a lot of data including learners' information, Web logs data, exercise/test scores, and questionnaire data were collected. These data were analyzed by the researchers.

\section{Characteristics}

Because the e-learning system is autonomous, learners can study knowledge about Chinese poetry at their own pace through browsing 'e-Tang'. There are some basic features involved in 'e-Tang' as follows:

- A fuzzy control algorithm is employed in order to arrange different learning courses for different learners. The learners' knowledge level varies greatly from learner to learner, but the current Webbased systems commonly arrange the same learning course for them without considering if learners' knowledge level and the difficulty level of the recommended course are matched to each other [2][5]. This issue is improved by employing the fuzzy control algorithm in this system. There are two variables in this algorithm, one is the scores of the learners (S), and the other is the difficulty of the learning course (D). Both of them are described by a fuzzy set $\{\mathrm{NB}, \mathrm{NS}, \mathrm{ZO}, \mathrm{PS}, \mathrm{PB}\}$. In the learners' score set, NB, NS, ZO, PS, PB are very bad, bad, moderate, good, and very good, respectively. In the same way, the difficulty of each learning course is described by the categories of very difficult, difficult, moderate, easy and very easy, which was mentioned as 5 ranks in Section 3.3. In reality, it is difficult to judge what kind of score is bad and what kind of learning course is difficult. All the judgments are based on the experiences of an instructor. Considering the number of questions in each exercise or test is only 5 or 10 in the 'e-Tang' system, the relationship among the fuzzy label and the two variables is shown in Table II. In addition, the fuzzy rules between the two variables are as follows:

If $\mathrm{S}=\mathrm{NB}$, then $\mathrm{D}=\mathrm{PB}$; or

If $\mathrm{S}=\mathrm{NS}$, then $\mathrm{D}=\mathrm{PS}$; or

If $\mathrm{S}=\mathrm{ZO}$, then $\mathrm{D}=\mathrm{ZO}$; or

If $\mathrm{S}=\mathrm{PS}$, then $\mathrm{D}=\mathrm{NS}$; or

If $\mathrm{S}=\mathrm{PB}$, then $\mathrm{D}=\mathrm{NB}$.

TABLE II. A LOOK-UP TABLE FOR FUZZY LABEL AND TWO CARIABLES

\begin{tabular}{|c|c|c|}
\hline Fuzzy label & The score of the learner & $\begin{array}{c}\text { The difficulty level of } \\
\text { learning course }\end{array}$ \\
\hline NB & $0 \leq S \leq 20$ & Rank 5 \\
\hline NS & $20<S \leq 40$ & Rank 4 \\
\hline ZO & $40<S \leq 60$ & Rank 3 \\
\hline PS & $60<S \leq 80$ & Rank 2 \\
\hline PB & $80<S \leq 100$ & Rank 1 \\
\hline
\end{tabular}

Thus, the different rank of each learning course can be arranged to the corresponding learners in accordance with their scores. Personalized learning performance can be promoted according to the fuzzy control algorithm.

- Abundant learning materials about Chinese poetry are provided to foreign learners. Via browsing these Web pages, learners are allowed to comprehend the evolution and characteristics of Chinese poetry, listen to the Chinese sound of the special poems, see the annotation of poetic words appearing as pop-up windows by placing the cursor on the text, and read the background of poetic writings, poet introductions, and so on.

Not only the reading materials, but also exercises and tests are provided to learners for assessing their learning. After completing the questions and submitting the answers online, learners receive a feedback page, including the score and the interpretation of answers from 'e-Tang'. Moreover, the score is recorded in the learners' information database. The learners' scores of exercise/test are considered to be one of the most important assessment factors for Chinese poetry learning.

- Effective interactive tools for foreign learners are provided in this system. There are two kinds of tools in this system, one is a search engine and the other is communication tools.

To search Chinese poetry on the Web, first of all researchers set up a Tang poems database. Since the quantity of Chinese poetry is so large, we have chosen 300 Tang Poems, one of the primers for Chinese poetry learning, and have put it in digital form. The whole 321 poems of this 
book are collected in the 'Tang poems database' which is arranged in the order of chapters. In addition, Chinese Pinyin, Chinese audio files, and English translations are added in the database. Therefore, learners can not only browse the contents of this book in the Web page according to the sequence of chapters, but also can search Tang poems they need by inputting title, poet, or other keywords.

In order to promote interaction between both teachers and learners, and learners and learners, communication tools such as a chat room, BBS, and e-mail are provided by the 'e-Tang' system. It enables learners to communicate with others and to construct their own knowledge.

The three characteristics of 'e-Tang' mentioned above are realized by employing Perl, one of the most popular scripting languages, which is used for creating dynamic Web pages and a lot of CGI programs.

\section{EvaluATION OF 'E-TANG'}

\section{A. Method}

'e-Tang' is designed and implemented in supporting Chinese poetry learning and facilitating active self-learning. To verify the efficiency of this system, a questionnaire survey on foreign learners was conducted online. The questionnaire, consisting of 13 five-point Likert scale items (Strongly Disagree, Disagree, Undecided, Agree, Strongly Agree), was developed and administered in Chinese and English. Those questionnaire items were composed of four subscales: (1) perceived ease of use; (2) perceived usefulness of contents; (3) perceived usefulness of tools and (4) learning satisfaction. The learners were asked to indicate the extent to which they agree or disagree with these items by marking one of the responses ranging from 'strongly disagree' to 'strongly agree'. After that, the learners were also asked to write their ideas and comments about 'e-Tang' freely in an open-ended question, which enabled us to know more about learners' attitude and reaction. Until now 19 completed questionnaires have been received.

\section{B. Results and Discussions}

As Table III shows, most of the learners think that the design of this system was friendly. And they have a positive attitude toward the ease of use of this system: 68\% (13 learners) think that it was easy to use this system.

TABLE III. PERCEIVED EASE OF USE

\begin{tabular}{|c|l|c|c|c|c|c|c|}
\hline$\#$ & \multicolumn{1}{|c|}{ Question } & SD & D & U & A & SA & Average \\
\hline 1 & $\begin{array}{l}\text { I think this system is } \\
\text { easy to use. }\end{array}$ & & 2 & 4 & 9 & 4 & 3.8 \\
\hline 2 & $\begin{array}{l}\text { I think the design of this } \\
\text { system is friendly. }\end{array}$ & & & & 11 & 8 & 4.4 \\
\hline
\end{tabular}

With respect to perceived usefulness of contents (as shown in Table IV), most of the learners did think that the contents of An introduction to Chinese poetry, Chinese poetry appreciation and exercises were useful during the online studying process, especially Chinese poetry appreciation, whose average evaluation was 4.3. Some of the learners said that listening to the Chinese sound of Tang poems made them feel interested and promoted their comprehend about the rhythm of Chinese poetry. However, 2 learners said that the questions in exercises were so detailed that they confused them and made them want to stop. To improve learners' interest and attract them, we must design more comprehensible questions in the exercise part.

TABLE IV. PERCEIVED USEFULNESS OF CONTENTS

\begin{tabular}{|c|l|c|c|c|c|c|c|}
\hline$\#$ & \multicolumn{1}{|c|}{ Question } & SD & D & U & A & SA & Average \\
\hline 3 & $\begin{array}{l}\text { I think An Introduction } \\
\text { to Chinese poetry was } \\
\text { useful during my online } \\
\text { studying process. }\end{array}$ & & 2 & 13 & 4 & 4.1 \\
\hline 4 & $\begin{array}{l}\text { I think Chinese poetry } \\
\text { Appreciation was useful } \\
\text { during my online } \\
\text { studying process. }\end{array}$ & $\begin{array}{l}\text { I think Exercise was } \\
\text { useful during my online } \\
\text { studying process. }\end{array}$ & 2 & 4 & 6 & 7 & 3.9 \\
\hline
\end{tabular}

According to Table $\mathrm{V}$, most of the learners agreed that the two kinds of tools (the search engine and communication tools) in 'e-Tang' were useful and effective in Chinese poetry learning. Moreover, learners wanted to choose a chat room in the three communication tools according to the questionnaire data, which verified that they prefer the synchronous communication mode to the asynchronous communication mode, and hope they can acquire just-in-time feedback.

TABLE V. PERCEIVED USEFULNESS OF CONTENTS

\begin{tabular}{|c|l|c|c|c|c|c|c|}
\hline$\#$ & \multicolumn{1}{|c|}{ Question } & SD & D & U & A & SA & Average \\
\hline 6 & $\begin{array}{l}\text { I think the search engine } \\
\text { was useful during my } \\
\text { online studying process. }\end{array}$ & $\begin{array}{l}\text { I think using chat room } \\
\text { to communicate was } \\
\text { useful during my online } \\
\text { studying process. }\end{array}$ & 2 & 9 & 8 & 4.3 \\
\hline 8 & $\begin{array}{l}\text { I think using BBS to } \\
\text { communicate was useful } \\
\text { during my online } \\
\text { studying process. }\end{array}$ & $\begin{array}{l}\text { I think using e-mail to } \\
\text { communicate was useful } \\
\text { during my online } \\
\text { studying process. }\end{array}$ & 2 & 9 & 6 & 6.2 \\
\hline With respect to learning & satisfaction as shown in Table \\
\hline
\end{tabular}

With respect to learning satisfaction as shown in Table VI, most of the learners think that the materials in this system did increase their interest, happiness, and achievements in the learning process, and they would like to continue using this system for Chinese poetry learning. In addition, $70 \%$ ( 7 learners) think that the materials in this system were enough 
TABLE VI. LEARNING SATISFACTION

\begin{tabular}{|c|l|c|c|c|c|c|c|}
\hline$\#$ & \multicolumn{1}{|c|}{ Question } & SD & D & U & A & SA & Average \\
\hline 10 & $\begin{array}{l}\text { The materials in this } \\
\text { system were enough for } \\
\text { Chinese poetry learning, } \\
\text { and did improve my } \\
\text { learning achievements. }\end{array}$ & 2 & 4 & 11 & 2 & 3.7 \\
\hline 11 & $\begin{array}{l}\text { The materials in this } \\
\text { system did increase my } \\
\text { interest of Chinese poetry. }\end{array}$ & $\begin{array}{l}\text { I got more happiness in } \\
\text { learning Chinese poetry } \\
\text { using this system. }\end{array}$ & & 4 & 9 & 6 & 4.1 \\
\hline 13 & $\begin{array}{l}\text { I will continue to use this } \\
\text { system for the study of } \\
\text { Chinese poetry. }\end{array}$ & 4 & 11 & 4 & 5 & 4.1 \\
\hline
\end{tabular}

for Chinese poetry learning.

In the open-ended question, learners showed their favor to this system by kindly stating some encouraging words to the researchers. Furthermore, they presented some useful suggestions about 'e-Tang', such as avoiding the misuse of BBS, and adopting clearer font sizes, and so on. Based on these suggestions, we have improved this system, for example, deleting the messages which are not related to Chinese poetry learning.

On the whole, according to the questionnaire, it is obvious that most learners believe that the 'e-Tang' system can effectively support and promote foreign learners to learn Chinese poetry actively and willingly.

\section{CONCLUSION}

In order to help foreign learners to learn Chinese poetry more effectively, a Chinese poetry learning support system named 'e-Tang' has been designed and implemented using Web technologies. This system provides abundant learning materials and effective interactive tools to learners, and a fuzzy control algorithm is employed to arrange different learning courses for different learners, thus personalizing learning performance. With this system, learners can study knowledge about Chinese poetry, do exercises, and communicate with others. For investigating learners' evaluations toward 'e-Tang' after using this system, a questionnaire survey on foreign learners was conducted online. The results of the questionnaire showed that most learners have positive attitudes toward the questions for all subscales (perceived ease of use, perceived usefulness of contents, perceived usefulness of tools, and learning satisfaction). That is, they did believe that the 'e-Tang' system can effectively support and promote Chinese poetry learning.

\section{ACKNOWLEDGMENT}

Supported by the Fundamental Research Funds for the Central Universities (Program No. CCNU12A03021)

\section{REFERENCES}

[1] Aihara, S.: "Learning Chinese from the base of pronunciation", Asahi press, 2003, pp.138-141.

[2] Chen, C. M., Lee, H. M., \& Chen, Y. H.: "Personalized e-learning system using item response theory". Computers and Education, 44(3), 2005, pp.237-255.

[3] Kusaka, T.: "The pronunciation of Chinese", Aruku press, 2007, pp.205-206.

[4] Liao, J. L.: "The investigation of Tang poetry learning in Japan", In Proceedings of the 5th meeting of the Japan Association of Chinese Language Education, 2007, pp.66.

[5] Papanikolaou, K. A., Grigoriadou, M., Magoulas, G. D., \& Kornilakis, H.: "Towards new forms of knowledge communication: The adaptive dimension of a Web-based learning environment". Computers and Education, 39, 2002, pp.333-360. 